\title{
Detecção de traços de narcisismo em conversas com predadores sexuais
}

\author{
Leonardo Ferreira dos Santos ${ }^{1}$, Gustavo Paiva Guedes ${ }^{1}$ \\ ${ }^{1}$ CEFET/RJ - Centro Federal de Educação Tecnológica Celso Suckow da Fonseca \\ Av. Maracana, 229 - Rio de Janeiro - RJ - Brasil. \\ leonardo.santos@eic.cefet-rj.br, gustavo.guedes@cefet-rj.br
}

\begin{abstract}
Sexual predators exploit the people to whom they relate in order to achieve their different individual goals. The profile of the sexual predator presents several mental disorders, among them, the narcissism stands out. In this scenario, the present work aims to detect traces of narcissism in the communication of sexual predators. To achieve this goal, we used the LIWC to analyze the use of personal pronouns in texts of these predators. In addition, a set of data from PAN-2012 is used. Results found present significant correlations between some categories related to personal pronouns and predatory conversations, consistent with results presented in the literature.
\end{abstract}

Resumo. Predadores sexuais exploram as pessoas com quem se relacionam a fim de atingir seus diferentes objetivos individuais. O perfil do predador sexual apresenta variados transtornos mentais, dentre eles, o destaca-se o narcisismo. Nesse cenário, o presente trabalho tem o objetivo de detectar traços de narcisismo na comunicação dos predadores sexuais. Para atingir esse objetivo, utilizamos o LIWC para analisar o uso de pronomes pessoais em textos desses predadores. Além disso, é utilizado um conjunto com os dados disponibilizados pela PAN-2012. Resultados encontrados apresentam correlações significativas entre algumas categorias relacionadas aos pronomes pessoais e conversas predatórias, coerentes com resultados apresentados na literatura.

\section{Introdução}

Segundo dados recentes, $9 \%$ dos menores de idade já sofreram algum tipo de abuso sexual na internet e, aproximadamente, 1 em cada 25 foi abordado por predadores sexuais para encontros fora do meio virtual ou contactados diretamente por meio de cartas, telefonemas ou in loco [Mitchell et al., 2014]. No entanto, esses números podem ser ainda maiores, visto que diversos casos de abusos sexuais em crianças não são reportados [Barbara et al., 2017]. Isso ocorre, primeiramente, porque crianças abusadas sexualmente tendem a ficar em silêncio, seja por culpa, vergonha ou ameaças [Bagley and King, 2003]. Além disso, o abuso sexual destrói a espontaneidade e a liberdade da criança, causando um terror solitário [Bagley and King, 2003].

Os predadores sexuais fazem uso de um padrão de comunicação enganosa que, inicialmente, busca criar uma falsa relação de confiança com o menor de idade para então, ter uma oportunidade de contato fora do meio virtual e executar o abuso sexual [Olson et al., 2007]. Comportamentos enganosos no meio virtual podem caracterizar a presença de transtornos mentais [Crossley, 2016]. Nesse cenário, algumas pesquisas na área de 
saúde têm estudado o comportamento dos usuários, o que vem permitido uma série de avanços na análise e compreensão de distúrbios mentais [Ayers et al., 2014]. Por exemplo, textos provenientes de salas de bate-papo e redes sociais têm sido objeto de estudo nos últimos anos, permitindo a identificação de transtornos mentais [Preoţiuc-Pietro et al., 2015; Coppersmith et al., 2014; Rodrigues et al., 2017].

O perfil do predador sexual apresenta variados transtornos mentais, dentre eles, o narcisismo [Crouch et al., 2015]. Algumas vezes definido, simplesmente, como alta autoestima [Gray, 2011], clinicamente o narcisismo é um transtorno de personalidade caracterizado por: acreditar ser "especial" e único; baixa empatia em relacionamentos e exploração das relações interpessoais com o objetivo de atender as próprias necessidades [Association et al., 2014].

O padrão de comunicação do agressor sexual e o caráter explorativo das relações interpessoais presentes no narcisismo definem a principal motivação para o presente trabalho, que tem como o principal objetivo a detecção traços de narcisismo em predadores sexuais. Para isso, é importante notar que a literatura destaca a existência de relação entre o narcisismo e utilização de pronomes pessoais [McGregor, 2010; Baryshevtsev and McGlone, 2018].

Até o momento dessa pesquisa não foram encontrados trabalhos com o objetivo de detectar traços de narcisismo na comunicação de predadores sexuais no meio virtual. Para realizar essa detecção, é empregado o dicionário linguístico LIWC que, com base na contagem de palavras presentes em textos, permite a extração de categorias psicolinguísticas [Pennebaker et al., 2001]. Os experimentos são realizados com os dados disponibilizados pela PAN-2012 ${ }^{1}$ para a competição de identificação de predadores sexuais.

As demais seções estão dispostas da seguinte maneira: na seção 2, são levantados trabalhos semelhantes e relacionados ao tema; na seção 3 é apresentado a metodologia para análise do conjunto de dados; na seção 4 são discutidos os resultados encontrados; por último, a seção 5 apresenta as conclusões, limitações e discussão sobre trabalhos futuros.

\section{Trabalhos relacionados}

O trabalho desenvolvido por Baryshevtsev and McGlone [2018] explorou a presença do uso de pronomes na comunicação de um predador sexual. Foram consideradas 561 transcrições de conversas ocorridas em meio virtual realizadas entre predadores sexuais e pseudo-vítimas (e.g., agentes federais agindo como menores de idade), disponibilizadas pelo site Perverted Justice ${ }^{2}$, em que foi possível constatar um uso maior de pronomes de segunda pessoa, comparado aos de primeira pessoa. Essa discrepância é justificada em [Olson et al., 2007], que indica que o objetivo de colocar a vítima como o foco da conversação é estabelecer uma relação de confiança, para que, em seguida, seja iniciado o abuso sexual, físico ou virtual.

No estudo apresentado em Drouin et al. [2017], os autores analisaram 590 conversas entre predadores sexuais e pseudo-vítimas, extraídas do site Perverted Justice ${ }^{2}$, com o objetivo de encontrar correlações com as seguintes categorias do LIWC: sexualidade,

\footnotetext{
${ }^{1}$ https://pan.webis.de/clef12/pan12-web/author-identification.html

${ }^{2}$ http://www.perverted-justice.com/
} 
influência e quantidade de palavras por sentença. Resultados apontaram que, perante suas pseudo-vítimas, predadores sexuais tendem a usar $91 \%$ mais palavras de cunho sexual, $66 \%$ mais palavras por sentença e $82 \%$ exerceram postura mais influente nas conversas quando comparado às pseudo-vítimas. Também foi destacada a utilidade do LIWC para o uso forense, permitindo uma análise objetiva de características psicológicas de predadores sexuais.

Em O'Reilly III et al. [2014], o narcisismo é identificado como uma característica existente nos líderes. Nesse estudo, o LIWC foi utilizado para analisar transcrições de teleconferências sobre lucros e cartas destinadas a acionistas. Foi possível identificar significativa correlação com o uso de pronomes pessoais, destacando-se pronomes de primeira pessoa.

Muitos trabalhos realizados buscam um melhor entendimento do perfil do agressor sexual em meio virtual, não somente psicológico mas também de atuação. No entanto, não foram encontrados estudos que busquem detectar traços de narcisismo em predadores sexuais em meio virtual. Sendo assim, o presente trabalho se enquadra nesse cenário, buscando contribuir com a detecção de traços de narcisismo em predadores sexuais com o uso do LIWC.

\section{Detecção de traços de narcisismo}

Esta seção descreve a metodologia utilizada para detectar a existência de traços de narcisismo em usuários predadores sexuais em comparação com usuários não-predadores. Para isso, são utilizados o conjunto de dados PAN-2012 e o dicionário do LIWC. Inicialmente, na subseção 3.1, são descritos o PAN-2012 e o LIWC. Em seguida, na subseção 3.2 , é descrita a metodologia utilizada nesse estudo.

\subsection{Conjuntos de dados}

PAN-2012: O conjunto de dados PAN-2012 consiste de conversas provenientes de alguns sites, dentre eles, o perverted-justice.com e irclog.org. Há um total de 66.914 conversas envolvendo 97.671 usuários únicos. Desses usuários, 142 foram rotulados como predadores. Existem 2.015 conversas em que os predadores estavam envolvidos. Nenhuma conversa possui mais de um predador e $68 \%$ das conversas continham dois usuários. O predador mais ativo se envolveu em 182 conversas.

LIWC: O Linguistic Inquiry and Word Count (LIWC) é um programa capaz de contabilizar palavras em diversas categorias psicolinguísticas [Pennebaker et al., 2001]. A versão do dicionário do LIWC utilizada nesse estudo possui mais de 6.400 palavras categorizadas em mais de 70 categorias [Pennebaker et al., 2015]. É interessante destacar que uma palavra pode estar associada a diversas categorias, por exemplo, a palavra amazing é categorizada como uma palavra afetiva e com emoção positiva.

\subsection{Metodologia}

A metodologia utilizada nesse estudo consiste em detectar a existência de traços de narcisismo - por meio da análise dos pronomes pessoais - em textos escritos por predadores sexuais. Isso é feito com a análise das diferenças existentes entre textos de predadores e texto de não-predadores. Os dados são provenientes do conjunto de dados PAN-2012 e as categorias utilizadas são oriundas do LIWC. A análise realizada é inspirada no estudo 
proposto por [McGregor, 2010]. Segundo esse estudo, (i) não existe correlação entre os narcisistas e a utilização de pronomes na primeira pessoa do plural (e.g., nós, nosso); (ii) não existe correlação entre o narcisismo e o uso de pronomes de segunda pessoa (e.g., você); (iii) não existe correlação entre o narcisismo e o uso de pronomes na terceira pessoa (e.g., ele).

Nesse cenário, o objetivo é utilizar o LIWC para avaliar as categorias de pronomes para evidenciar a diferença de uso pronominal entre predadores e não-predadores. Isso é efetuado com base na equação que define o tamanho de efeito $d$ de Cohen [Rosnow and Rosenthal, 1996], representada na Equação 1. As categorias do LIWC são representadas por $(0 \leq i<5)$, em que $i$ representa uma das cinco categorias de pronomes pessoais presentes no LIWC: $i$, we, you, shehe e they, representando pronomes na $1^{a}$ pessoa do singular, $1^{a}$ pessoa do plural, $2^{a}$ pessoa, $3^{a}$ pessoa do singular e $3^{a}$ pessoa do plural, respectivamente.

A média simples $\bar{X}_{P}^{i}$ é dada pela i-ésima componente do vetor de frequência dos textos dos predadores. Analogamente, $\bar{X}_{N P}^{i}$ representa a média simples da i-ésima componente do vetor de frequência dos textos dos não-predadores. Observando o denominador, $S D_{P}^{i}$ e $S D_{N P}^{i}$ representam os desvios padrão da i-ésima componente do vetor de frequência dos textos dos predadores e não-predadores, respectivamente. Valores positivos de $d_{i}$ indicam que mais palavras na categoria $i$ foram utilizadas pelos predadores e valores negativos, pelos não-predadores. Os valores de referência de $d$ são considerados a partir de [Cohen, 1988], em que $d=0.20$ indica um pequeno efeito, $d=0.50$ um médio efeito e $d=0.80$ um grande efeito.

$$
d_{i}=\frac{\bar{X}_{P}^{i}-\bar{X}_{N P}^{i}}{\sqrt{\left(\left(S D_{P}^{i}\right)^{2}+\left(S D_{N P}^{i}\right)^{2}\right) / 2}}
$$

\section{Resultados}

Nesse estudo, são exibidos os resultados encontrados para a diferença entre a utilização de pronomes pessoais por predadores sexuais e não-predadores. Para isso, é exibido o tamanho de efeito $d$ de Cohen para cada classe de pronomes do LIWC. Além disso, para ambos os grupos, são apresentados os valores para a média e o desvio padrão. A Tabela 1 apresenta os resultados encontrados.

Tabela 1. Tamanho do efeito $d$ de Cohen em cada uma das classe de pronomes do LIWC. Os resultados são apresentados na forma de média (M), desvio padrão (D) e (d) para o tamanho de efeito.

\begin{tabular}{|c|c|c|c|c|c|c|}
\hline \multirow[b]{2}{*}{ Dimensão } & \multirow[b]{2}{*}{ Exemplo } & \multicolumn{2}{|c|}{ Predadores Sexuais } & \multicolumn{2}{|c|}{ Não-predadores } & \multirow[b]{2}{*}{$(d)$} \\
\hline & & $\mathrm{M}$ & $\mathrm{D}$ & $\mathrm{M}$ & $\mathrm{D}$ & \\
\hline $\mathrm{i}$ & $I$, mine & 6.65 & 6.38 & 3.17 & 5.00 & 0.61 \\
\hline we & we, our & 0.31 & 1.21 & 0.24 & 1.41 & 0.06 \\
\hline you & you, your & 10.74 & 10.90 & 4.20 & 6.49 & 0.73 \\
\hline shehe & her, him & 0.22 & 0.76 & 0.17 & 1.35 & 0.05 \\
\hline they & they, their & 0.15 & 0.67 & 0.13 & 0.72 & 0.03 \\
\hline
\end{tabular}


Os valores em em negrito destacam valores significantes de tamanho de efeito. Pode-se evidenciar um tamanho de efeito médio no uso de pronomes na primeira pessoa (i.e., 0.61) e no uso de pronomes na segunda pessoa (i.e., 0.73). Para os demais pronomes não foram encontrados valores de efeito significantes.

\section{Discussão}

O estudo aqui apresentado objetivou detectar traços de narcisismo em indivíduos identificados como predadores sexuais. Essa tarefa foi fundamentada na premissa de que esses predadores tendem a ser narcisistas. Para efetuar esse estudo, foi utilizado um conjunto de dados de predadores sexuais, denominado PAN-2012.

Os resultados encontrados corroboram com duas conclusões apresentadas em [McGregor, 2010]: (i) não existe correlação entre os narcisistas e a utilização de pronomes na primeira pessoa do plural (e.g., nós, nosso); (iii) não existe correlação entre o narcisismo e o uso de pronomes na terceira pessoa. Isso pôde ser evidenciado pelos tamanhos de efeito apresentados para a categoria de pronomes pessoais na primeira pessoa e na terceira pessoa.

Porém, nosso estudo encontrou um tamanho médio de efeito para os pronomes da terceira pessoa, não apresentando o mesmo resultado encontrado em [McGregor, 2010] que destaca que (ii) não existe correlação entre o narcisismo e o uso de pronomes na segunda pessoa. Entretanto, conforme evidenciado em estudo recente, indivíduos que interagem de forma online com menores de idade, com objetivos de encontrá-los pessoalmente, tendem a utilizar mais pronomes na segunda pessoa do singular, indicando que isso pode ser uma maneira de transmitir interesse e atenção à criança [Baryshevtsev and McGlone, 2018]. O estudo ainda destaca que focar mais na criança pode transmitir cuidado e preocupação (e.g., "você é melhor que isso"), resultando em uma maior receptividade por parte da criança.

Embora esse estudo ofereça resultados preliminares, é interessante destacar que todos os resultados encontrados foram coerentes com resultados apresentados na literatura. Para trabalhos futuros, é considerada a inclusão de outras categorias do LIWC para análise no conjunto de dados usado no presente artigo. Um bom exemplo seria a utilização de palavras de cunho sexual e xingamentos.

\section{Referências}

Association, A. P. et al. (2014). DSM-5: Manual diagnóstico e estatístico de transtornos mentais. Artmed Editora.

Ayers, J. W., Althouse, B. M., and Dredze, M. (2014). Could behavioral medicine lead the web data revolution? Jama, 311(14):1399-1400.

Bagley, C. and King, K. (2003). Child sexual abuse: The search for healing. Routledge.

Barbara, G., Collini, F., Cattaneo, C., Facchin, F., Vercellini, P., Chiappa, L., and Kustermann, A. (2017). Sexual violence against adolescent girls: labeling it to avoid normalization. Journal of Women's Health, 26(11):1146-1149.

Baryshevtsev, M. V. and McGlone, M. S. (2018). Pronoun usage in online sexual predation. Cyberpsychology, Behavior, and Social Networking, 21(2):117-122.

Cohen, J. (1988). Statistical power analysis for the behavioral sciences 2nd edn. 
Coppersmith, G., Dredze, M., and Harman, C. (2014). Quantifying mental health signals in twitter. In Proceedings of the Workshop on Computational Linguistics and Clinical Psychology: From Linguistic Signal to Clinical Reality, pages 51-60.

Crossley, L. N. (2016). The influence of the Dark Triad and communication medium on deceptive outcomes. $\mathrm{PhD}$ thesis, University of British Columbia.

Crouch, J. L., Hiraoka, R., Rutledge, E., Zengel, B., Skowronski, J. J., and Milner, J. S. (2015). Is narcissism associated with child physical abuse risk? Journal of Family Violence, 30(3):373-380.

Drouin, M., Boyd, R. L., Hancock, J. T., and James, A. (2017). Linguistic analysis of chat transcripts from child predator undercover sex stings. The Journal of Forensic Psychiatry \& Psychology, 28(4):437-457.

Gray, P. (2011). The decline of play and the rise of psychopathology in children and adolescents. American Journal of Play, 3(4):443-463.

McGregor, S. (2010). The analysis of personality through language: Narcissism predicts use of shame-related words in narratives.

Mitchell, K., Jones, L., Finkelhor, D., and Wolak, J. (2014). Trends in unwanted sexual solicitations: Findings from the youth internet safety studies. Youth Internet Safety Survey Bulletin.

Olson, L. N., Daggs, J. L., Ellevold, B. L., and Rogers, T. K. (2007). Entrapping the innocent: Toward a theory of child sexual predators' luring communication. Communication Theory, 17(3):231-251.

O'Reilly III, C. A., Doerr, B., Caldwell, D. F., and Chatman, J. A. (2014). Narcissistic ceos and executive compensation. The Leadership Quarterly, 25(2):218-231.

Pennebaker, J. W., Boyd, R. L., Jordan, K., and Blackburn, K. (2015). The development and psychometric properties of liwc2015. Technical report.

Pennebaker, J. W., Francis, M. E., and Booth, R. J. (2001). Linguistic inquiry and word count: Liwc 2001. Mahway: Lawrence Erlbaum Associates, 71(2001):2001.

Preoţiuc-Pietro, D., Eichstaedt, J., Park, G., Sap, M., Smith, L., Tobolsky, V., Schwartz, H. A., and Ungar, L. (2015). The role of personality, age, and gender in tweeting about mental illness. In Proceedings of the 2nd Workshop on Computational Linguistics and Clinical Psychology: From Linguistic Signal to Clinical Reality, pages 21-30.

Rodrigues, R. G. R., Pereira, W. W. P., Bezerra, E. B., and Guedes, G. P. G. (2017). Inferência de idade utilizando o liwc: identificando potenciais predadores sexuais. In Proceedings of the Brazilian Workshop on Social Network Analysis and Mining. SBC.

Rosnow, R. L. and Rosenthal, R. (1996). Computing contrasts, effect sizes, and counternulls on other people's published data: General procedures for research consumers. Psychological Methods, 1(4):331. 